\title{
Exciton-Polariton Gas as a Nonequilibrium Coolant
}

\author{
Sebastian Klembt, ${ }^{1}$ Emilien Durupt, ${ }^{1}$ Sanjoy Datta, ${ }^{2}$ Thorsten Klein, ${ }^{3}$ Augustin Baas, ${ }^{1}$ Yoan Léger, ${ }^{4}$ Carsten Kruse, ${ }^{3}$ \\ Detlef Hommel, ${ }^{3}$ Anna Minguzzi, ${ }^{2}$ and Maxime Richard ${ }^{1}$ \\ ${ }^{1}$ Institut Néel, Université Grenoble Alpes and CNRS, B.P. 166, 38042 Grenoble, France \\ ${ }^{2}$ LPMMC, Université Grenoble Alpes and CNRS, B.P. 166, 38042 Grenoble, France \\ ${ }^{3}$ University of Bremen, P.O. Box 330440, 28334 Bremen, Germany \\ ${ }^{4}$ Laboratoire FOTON, CNRS, INSA de Rennes, 35708 Rennes, France
}

(Received 8 December 2014; revised manuscript received 4 February 2015; published 5 May 2015)

Using angle-resolved Raman spectroscopy, we show that a resonantly excited ground-state excitonpolariton fluid behaves like a nonequilibrium coolant for its host solid-state semiconductor microcavity. With this optical technique, we obtain a detailed measurement of the thermal fluxes generated by the pumped polaritons. We thus find a maximum cooling power for a cryostat temperature of $50 \mathrm{~K}$ and below where optical cooling is usually suppressed, and we identify the participation of an ultrafast cooling mechanism. We also show that the nonequilibrium character of polaritons constitutes an unexpected resource: each scattering event can remove more heat from the solid than would be normally allowed using a thermal fluid with normal internal equilibration.

DOI: 10.1103/PhysRevLett.114.186403

PACS numbers: 71.36.+c, 78.20.nd, 78.30.Fs

Owing to the selection rules in light-matter interaction, light can pick up energy and momentum from matter and conversely, as soon as translational invariance is broken by, e.g., a dielectric interface or a pointlike dipole such as an atom. This seemingly trivial property is at the basis of several spectacular achievements such as Doppler cooling of atomic gases [1], optical tweezers [2], or cavity mirror motion cooling by radiation pressure [3-5]. Long before such ideas were implemented, Pringsheim proposed in 1929 that such an effect could be used to cool down solids [6]. Indeed, discrete translational invariance in ideal solids is broken by thermal vibrations of the lattice (phonons), the energy of which can be transferred to light. Such a mechanism is known nowadays as anti-Stokes fluorescence (ASF): a laser is tuned to the vibrational ground state of an electronic transition, such that the reemitted photons are likely to take away with them the energy of one or several thermal phonons, resulting in a temperature drop of the material. The technologic advantages of such a technique are obvious, like the absence of moving mechanical parts, and the very convenient nature of light as a coolant, easy to create, dissipate, and propagate across large distances.

In order to implement this strategy, the material must feature an electronic transition with a high radiative rate and quantum efficiency (i.e., a vanishing nonradiative recombination). Owing to their imperfections, these conditions are hard to meet in real materials. The best results so far have been obtained in ytterbium-doped crystals. Indeed, in spite

Published by the American Physical Society under the terms of the Creative Commons Attribution 3.0 License. Further distribution of this work must maintain attribution to the author(s) and the published article's title, journal citation, and DOI. of the long radiative lifetime and weak oscillator strength of the embedded ytterbium atoms, their high quantum efficiency allowed cooling from room temperature down to $T=110 \mathrm{~K}[7,8]$. In semiconductor materials, the excitonic transition has a much larger oscillator strength, a stronger coupling to phonons, and a shorter radiative rate. It was thus predicted that optical cooling in semiconductors should be more efficient $[9,10]$. However, despite encouraging results $[11,12]$, it is only recently that a room temperature $\mathrm{ZnS}$ nanostructure could be cooled down to $T=260 \mathrm{~K}$ [13]. The difficulty lies in the fact that, owing to their extended nature, excitons are more prone to multiphonon nonradiative relaxation via impurity or defect states. These relaxation channels involve phonon cascade emission [14] that competes with the phonon absorption achieved by ASF, making it hard to reach a positive net cooling power.

In this work, instead of bare photons, we consider using resonant optical pumping of ground state exciton polaritons to excite polariton ASF, and thus generate a cooling power in a solid-state semiconductor microcavity (MC) in the strong coupling regime [cf. Fig. 1(a)]. Polaritons are bidimensional quasiparticles that benefit from a halfphotonic, half-excitonic nature [15] to achieve much stronger interaction with phonons than photons $[16,17]$. Figure 1(c) shows the principle of the most interesting cooling mechanism achieved by this excitation scheme, and that we report in this Letter: within a short lifetime of $\sim 1 \mathrm{ps}$, a polariton can be excited into a higher energy state by absorbing a thermal acoustic phonon of average energy $\hbar \Omega_{f}$. Its subsequent radiative recombination results in the net energy transfer of $\hbar \Omega_{f}$ from the thermal phonon bath to the outside electromagnetic vacuum. This mechanism is illustrated in Fig. 1(b) in terms of nonequilibrium 
(a)
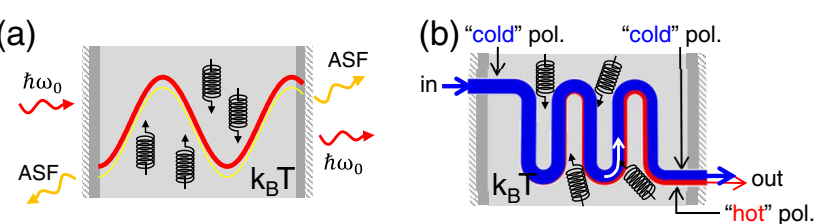

(c) Fast cooling

(d) Slow cooling

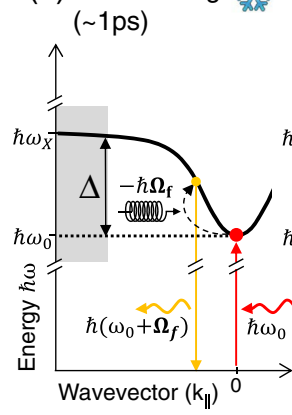
( 200ps)

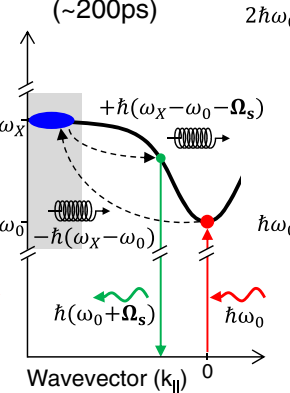

$\uparrow(\mathrm{e})$ Heating

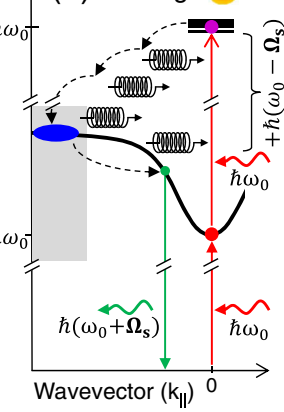

FIG. 1 (color online). (a) Principle of the cooling method: the polariton field absorbs thermal phonons resulting in polariton ASF emission. The wavy arrows depict photons, while the springlike arrows represent phonons. (b) Illustration of the cooling mechanism in terms of nonequilibrium thermodynamics: the blue (red) intracavity flow represents "cold" ("hot") polaritons. (c),(d), and (e) Summarize the three heat exchange mechanisms set in motion by the cooling method. Phonon energy is counted positive (negative) for emission (absorption). Fast cooling mechanism (b) removes phonons of average energy $\hbar \Omega_{f}$, slow cooling mechanism (c) removes phonons of average energy $\hbar \Omega_{s}$, while two-photon absorption (d) generates a phonon cascade (heat) of average energy $\hbar \omega_{0}-\hbar \Omega_{s}$. The gray rectangle is the area outside the polariton light cone.

thermodynamics: "cold" polaritons are injected into the microcavity; over time a fraction of them picks up heat from the surrounding phonon bath, giving rise to a "hot" component of the polariton fluid. Hot and cold polaritons finally exit the microcavity under the form of photons. Two additional thermal exchange mechanisms also take place, a cooling one shown in Fig. 1(d), and a heating one shown in Fig. 1(e), as will be discussed later.

Polaritons have many advantages over other optical methods to cool down semiconductor materials: their cavity-like dispersion [cf. $\omega\left(k_{\|}\right)$in Figs. 1(c)-1(e)] fully inhibits the Stokes emission, which is a source of phonon emission. Moreover, owing to their bidimensional degree of freedom, a 2D continuum of states is available for antiStokes scattering, so that thermal phonons of arbitrary low energy can be removed in the process, thus preventing a cooling power cutoff at low temperatures. Another useful property of polaritons in this context is their ultralight mass of $\sim 10^{-4}$ in electron mass unit: it allows anti-Stokes polaritons to remain within the light cone even when high energy thermal phonons are involved [cf. Fig. 1(c)], and it quenches the coupling with pointlike defects that could cause nonradiative recombination. We verified indeed that this relaxation channel could be safely neglected in this thermal analysis [18]. Finally, the strong coupling regime

reduces the scattering of polaritons towards the long-lived dark exciton level [27] that favors nonradiative recombination. In this context, a large Rabi splitting is desirable. We thus fabricated a high-quality selenide-based MC for our experiment similar to that used in Ref. [28], displaying a Rabi splitting of $\hbar \Omega_{R}=29 \mathrm{meV}$, stable for any cryostat temperature ranging from $T=5$ to $T=150 \mathrm{~K}$. In this experiment, polariton cooling is set in motion by shining a linearly polarized $\mathrm{cw}$ laser beam at normal incidence on the $\mathrm{MC}$, focused in a spatially Fourier-transform spot of $20 \mu \mathrm{m}$ diameter, and resonant with $k_{\|}=\sqrt{k_{x}^{2}+k_{y}^{2}}=0$ polaritons at $\hbar \omega_{0}=2791.1 \mathrm{meV}$.

Our aim is not to directly measure a temperature drop of the MC. Such a task is indeed technologically challenging: one first needs to grow a microcavity with a nonabsorbing substrate on the back side, or needs to remove it; second, suspended microstructures must then be designed and etched in order to achieve the best possible thermal insulation from the rest of the sample. In this work, in order to demonstrate the principle and to characterize the performance of this cooling method, we use polariton ASF to perform a detailed measurement of the different thermal fluxes generated within the MC by the pumped polaritons. Since polariton ASF is isotropic and depolarized by antiStokes scattering [18], it is measured by angle-resolved, cross-polarized spectroscopy in the reflection configuration. We choose a negative cavity-exciton energy detuning $\delta=-17 \mathrm{meV}$ (at $T=4.2 \mathrm{~K}$ ) of the MC so that the polariton dispersion spans over an energy range of $\Delta=$ $\hbar \omega_{X}-\hbar \omega_{0}=26 \mathrm{meV}$ [cf. Fig. 1(c)]. In this way, at the temperature we are interested in, most of the thermal phonons have an energy lower than $\Delta$ and thus create anti-Stokes polaritons within the light cone. The small remaining fraction creates bare excitons at large momenta as shown in Fig. 1(d). These two mechanisms will be labeled as fast and slow cooling, respectively, thereafter.

A typical raw ASF spectrum is shown in Fig. 2(a) for a cryostat temperature of $T=20 \mathrm{~K}$ in logarithmic color scale. It is obtained by spectrally resolving a narrow slice (along $k_{y}$ ) of the ASF emission, where residual laser light is further rejected by spectral and $k$-space filtering [crosshatched region at the bottom of Fig. 2(a)]. A careful calibration of the whole setup optical transmission allows us to obtain the ASF spectrum $i_{\mathrm{ASF}}(\omega)$ in absolute antiStokes photon emission rate in counts per second. Finally, since ASF emission is isotropic, the total ASF spectral density $I_{\mathrm{ASF}}(\omega)$ within a cone of $8.4^{\circ} \leq \theta \leq 43^{\circ}$ radius is extrapolated. $I_{\mathrm{ASF}}(\omega)$ normalized by the excitation power $P_{\text {las }}$ is plotted in Fig. 2(b) for different $P_{\text {las }}$. Details on the experimental setup and data treatment are given in the Supplemental Material [18].

A first striking behavior is that the high energy part of the spectra behaves nonlinearly with respect to $P_{\text {las }}$. A careful numerical analysis of this data set allows us to extract $A^{(1)}(\omega)$ and $A^{(2)}(\omega)$, i.e., the fraction of the ASF spectrum 
(a) Emission angle (deg.)

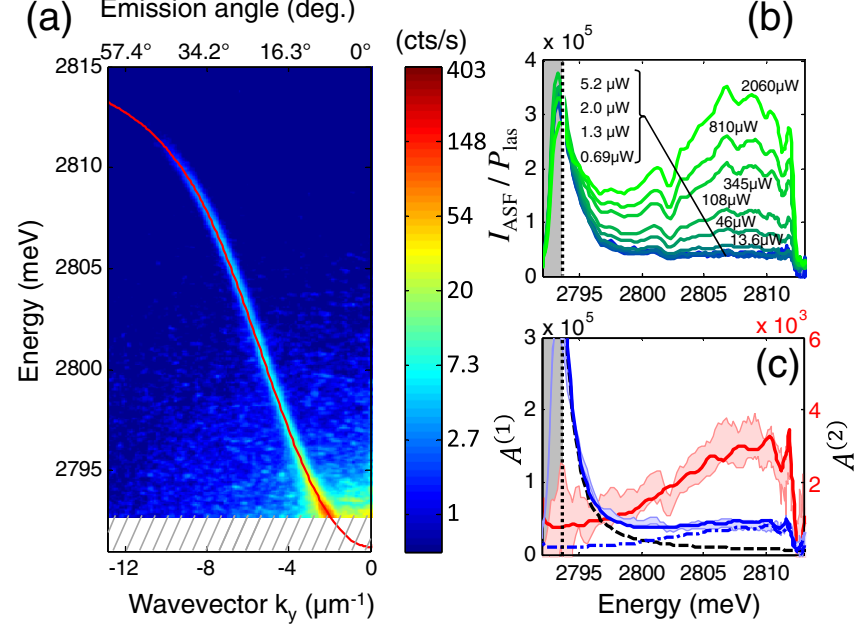

FIG. 2 (color online). (a) ASF spectrum at $T=20 \mathrm{~K}$, in logarithmic color scale versus $k_{y}$ and $\hbar \omega$. The corresponding emission angle $\theta$ is shown on the top axis. The red line is a fit to the dispersion shape. (b) ASF spectral density divided by $P_{\text {las }}$ in $\mathrm{cts} / \mathrm{s} / \mathrm{meV} / \mu \mathrm{W}$. The color of the solid lines evolves from blue to green for increasing laser power $P_{\text {las }}$. (c) Measured (blue stripe) and fitted (solid blue line) linear component $A^{(1)}(\omega)$ of the spectrum in cts $/ \mathrm{s} / \mathrm{meV} / \mu \mathrm{W}$. Red: measured (red stripe) and fitted (solid red line) quadratic component of the spectrum in cts $/ \mathrm{s} / \mathrm{meV} / \mu \mathrm{W}^{2}$. The solid red line is proportional to $I_{\mathrm{PL}}(\omega)$. The dashed black line is the fast cooling component in $A^{(1)}(\omega)$, while the dash-dotted blue line is the slow one.

that behaves linearly and quadratically, respectively, with respect to $P_{\text {las }}$ [18]. As shown in Fig. 2(c), $A^{(2)}(\omega)$ has the same spectral shape as a photoluminescence spectrum $I_{\mathrm{PL}}(\omega)$ [solid red line in Fig. 2(c)] obtained under nonresonant excitation with a cw laser tuned at $\hbar \omega_{\mathrm{nr}}=$ $2990 \mathrm{meV}$, i.e., above the band to band transition. $A^{(2)}(\omega)$ thus clearly results from two-photon absorption of the pump laser under the form of high energy free carriers, followed by a phonon cascade that relaxes the carriers back into the polariton states [i.e., the mechanism shown in Fig. 1(e)]. From the thermal balance point of view, this mechanism is a source of heat since each absorption event adds a cascade of phonons of total average energy $\hbar\left(\omega_{0}-\Omega_{s}\right)$ into the lattice, where $\hbar\left(\omega_{0}+\Omega_{s}\right)$ is the average energy of $I_{\mathrm{PL}}(\omega)$. Fortunately, its quadratic behavior with respect to $P_{\text {las }}$ makes its contribution negligible at low enough excitation power $P_{\text {las }}$.

$A^{(1)}(\omega)$, the part of the spectrum that is linear with $P_{\text {las }}$, results from the absorption of thermal phonons by ground state polaritons of energy $\hbar \omega_{0}$ followed by fluorescence at higher energy. It thus provides the wanted cooling power, and involves two distinct mechanisms. In the first one, that we call "fast," a ground state polariton is scattered directly into an excited polariton of average energy $\hbar\left(\omega_{0}+\Omega_{f}\right)$, that recombines radiatively within a time scale of $\sim 1 \mathrm{ps}$ [cf. Fig. 1(c)]. The second one, that we call "slow," has the excitonic reservoir (outside the light cone) as a longer lifetime ( 200 ps [29]) intermediate state [Fig. 1(d)].
Within a spectral analysis of $A^{(1)}(\omega)$, the proportion of these two different contributions can be evaluated quantitatively. Indeed, a Fermi golden rule approach provides an accurate simulation of the fast ASF spectrum $I_{\text {th }}(\omega)$ [dashed line in Fig. 2(c)] that shows that its contribution is maximum at low energy (only the high energy flank is visible experimentally). On the other hand, the slow ASF spectrum is well accounted for by the experimentally measured nonresonant spectrum $I_{\mathrm{PL}}(\omega)$, which exhibits a pronounced bottleneck $\sim 15 \mathrm{meV}$ above the ground state [dash-dotted blue line in Fig. 2(c)]. We clearly see in $A^{(1)}(\omega)$ these two contributions: the upper flank of a peak on the low energy side energy (fast ASF) and a shoulder on the high energy side (slow ASF). Using both $I_{\text {th }}(\omega)$ and $I_{\mathrm{PL}}(\omega)$ to fit $A^{(1)}(\omega)$ [cf. blue solid line in Fig. 2(c)], the fraction of fast cooling $\rho$ contributing to the overall cooling power is thus evaluated. The accuracy of this procedure is assured by the fact that $I_{\mathrm{th}}(\omega)$ and $I_{\mathrm{PL}}(\omega)$ have a quite different spectral shape. Note that since the microcavity parameters, $I_{\mathrm{PL}}(\omega)$, as well as the materials parameters are measured or known from the literature (cf. Supplemental Material [18]) only the overall multiplicative factors of $A^{(1)}(\omega)$ and $A^{(2)}(\omega)$ are free fitting parameters.

We have carried out this analysis at different cryostat temperatures. We found that the fast cooling mechanism has a peak contribution of $\rho=61 \%$ at $T=20 \mathrm{~K}$, and remains significant up to $T \simeq 100 \mathrm{~K}$ [cf. Fig. 3(b)]. This fast cooling mechanism is unique to polaritons: its time scale is fixed by the polariton lifetime which is 2 orders of magnitude shorter than the slow cooling one. This dynamics is even faster than the phonons typical thermalization time [30]. The observation of this fast cooling mechanism in a $\mathrm{MC}$ in the strong coupling regime is the key result of this Letter. The slow cooling mechanism is reminiscent from that involved in exciton-enhanced optical cooling [9]. At such low temperatures, its contribution can seem surprising as it involves thermal phonons of energy comparable with $\Delta \gg k_{B} T$. However, the weak phonon population at this energy is compensated by two aspects: (i) the excitonic density of states, which is 4 orders of magnitude larger than that of polaritons, and (ii) the fact that for such large $\Delta$ 's the phonons involved are of the longitudinal optical (LO) type, and thus exhibit a coupling strength with polaritons 50 times larger than the acoustic ones involved in the fast cooling [31].

Now that we have a measurement of the three main mechanisms involved in the heat exchange between polariton and thermal phonons, we can derive an experimental value of the thermal energy removed from the MC per units of time [i.e., the cooling or fridge (fr) power] as

$$
\begin{aligned}
P_{\mathrm{fr}}\left(P_{\mathrm{las}}, T\right)= & \int d \omega\left\{P_{\mathrm{las}} \hbar\left(\omega-\omega_{0}\right) A^{(1)}(\omega, T)\right. \\
& \left.-P_{\mathrm{las}}^{2} \hbar\left(2 \omega_{0}-\omega\right) A^{(2)}(\omega, T)\right\},
\end{aligned}
$$


(a)

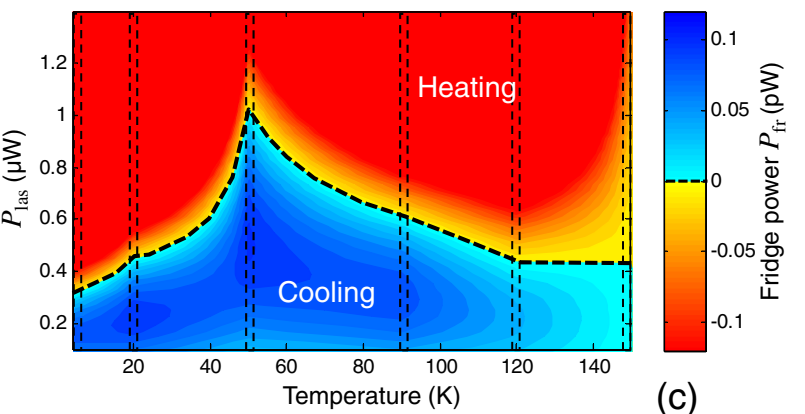

(b)
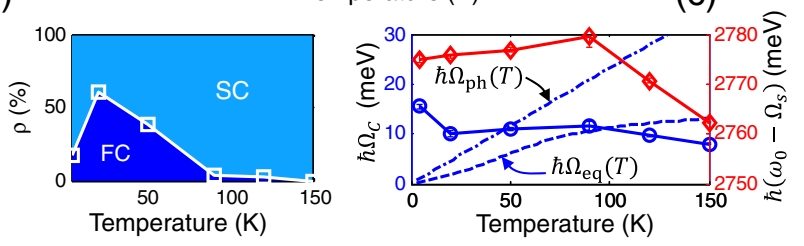

FIG. 3 (color online). (a) Total cooling power $P_{\mathrm{fr}}$ versus cryostat temperature (T) and laser power $P_{\text {las }}$. The dashed vertical rectangles contain color plots of the measured function $P_{\mathrm{fr}}\left(P_{\text {las }}\right)$ [see main text and Eq. (1) for the method]. The rest of the color plot is an interpolation serving as a guide to the eye. The dashed line separates the cooling region from the heating one. (b) Participation $\rho$ of fast cooling (FC) (SC stands for slow cooling) involved in the overall cooling process. (c) Red crosses: average energy $\hbar \omega_{0}-\hbar \Omega_{s}$ of the phonon cascade involved in the twophoton absorption heating. Blue hollow circles: average phonon energy $\hbar \Omega_{c}$ removed by the overall cooling process. The dashed line shows the theoretical average energy of polaritons $\hbar \Omega_{\mathrm{eq}}(T)$ assuming thermal equilibrium with the phonon bath (same temperature $T) . \hbar \Omega_{\mathrm{ph}}(T)$ is the average energy of thermal phonons.

where $\hbar\left(\omega-\omega_{0}\right)$ is the energy of a phonon which has been removed when a photon is detected at the energy $\hbar \omega$. Positive $P_{\text {fr }}$ means cooling while negative means heating. Note that in this approach, $P_{\mathrm{fr}}$ is a lower bound of the true cooling power since due to the objective finite numerical aperture, only $46 \%$ of the whole momentum space is accessible. Indeed, the inaccessible large $k_{\|}$ring involves the absorption of high energy thermal phonons, which have a large contribution to cooling via the slow channel. We extracted $P_{\text {fr }}$ for temperatures ranging from $T=4.2$ to $T=150 \mathrm{~K}$. The result is summarized in Fig. 3(a) versus temperature and laser power. We find that the maximum cooling power $P_{\mathrm{fr}}^{\max }=(0.10 \pm 0.02) \mathrm{pW}$, with $\rho=40 \%$ is achieved at $T=50 \mathrm{~K}$, and remains positive below. As explained above, this is only possible because polariton ASF does not involve a discontinuous electronic density of states but rather a continuous one with no energy gap between the pump polaritons and the available anti-Stokes states. Therefore, phonons of very low energy can be pumped out from the thermal bath by this method. Such a behavior is another unique feature of polariton cooling and constitutes the second key result of this Letter. Note that this cooling power is generated within a micron-scale volume resulting in a large cooling power density of $p_{\text {fr }}=(80 \pm 16) \mu \mathrm{W} \mathrm{cm}^{-3}$. Finally, we see in Fig. 3(c) (red diamond) why the main limitation to polariton cooling is two-photon absorption; indeed, although this absorption rate is low as compared to the cooling rate (at least at power $P_{\text {las }}$ low enough), each such event releases a large amount of heat $\hbar\left(\omega_{0}-\Omega_{s}\right)$ in average, ranging from 2760 to $2780 \mathrm{meV}$.

According to these results and our understanding of polariton cooling, both the fast cooling participation ratio $\rho$ and the cooling power $P_{\text {fr }}$ could be largely increased by making two fairly simple changes in the MC design. First, by lowering the quality factor $Q=\omega_{0} /(2 \pi \gamma)$ (presently, $Q=5600$ ), the ratio of the two-photon absorption rate over the cooling rate would decrease since the earlier scales as $1 / \gamma^{2}$ and the latter scales as $1 / \gamma$. Obviously, $Q$ cannot be decreased to arbitrarily low values as it must remain large enough for the strong coupling regime to be preserved. Second, when $\Delta$ exceeds the energy $\hbar \Omega_{\mathrm{LO}}^{\max }$ of the highest frequency LO phonon mode, the scattering of a polariton into the excitonic reservoir by a single phonon becomes forbidden by energy conservation. A strong suppression of the slow cooling mechanism in favor of the fast one is thus achieved. Such a suppression has been demonstrated already in a different context in a $\mathrm{ZnO} \mathrm{MC}$ [32]. An additional condition required to preserve the fast cooling mechanism is that the excitonic fraction should remain significant. Both conditions are easily met in state-of-theart selenide and telluride microcavities that combine large Rabi splitting and low LO phonon energy.

This work also gives us a striking insight on the thermal properties of a nonequilibrium cryogenic fluid (polaritons) interacting with a thermal bath (phonons) over a time scale too short for it to thermalize. As illustrated in Fig. 1(b), during their intracavity lifetime, the cold polaritons are heated up by the thermal phonons. The steady-state polariton gas resulting from this interaction is highly nonthermal -it consists of two independent components: a cold gas that did not interact with phonons and recombines at the same energy at which it entered the MC, and a smaller hot one, in which the captured heat is distributed according to a nonthermal distribution function, a measurement of which is given by $A^{(1)}(\omega)$. These hot and cold components do not mix up like in a normal fluid, because at the low densities involved in this cooling scheme, polaritons do not interact with each other. The properties of this hot component are rather unusual: in Fig. 3(c) we plotted $\hbar \Omega_{c}(T)$, the average energy of $A^{(1)}(\omega)$, which represents the average energy removed from the solid per scattering event. $\hbar \Omega_{\mathrm{eq}}(T)$, the average energy of a hypothetical polariton gas at thermal equilibrium is shown on the same plot. We find that between $T=4.2$ and $T=100 \mathrm{~K}, \hbar \Omega_{c}>\hbar \Omega_{\text {eq }}$, meaning that the thermal energy removed from the solid by each polariton of the hot component is larger than if they were at thermal equilibrium with the lattice. We can also compare $\hbar \Omega_{c}(T)$ with $\hbar \Omega_{\mathrm{ph}}(T) \simeq 2.701 k_{B} T$, the average energy of 
the thermal phonon bath. It appears that between $T=4.2$ and $T=50 \mathrm{~K}, \hbar \Omega_{c}>\hbar \Omega_{\mathrm{ph}}$, meaning this time that the hot component of the polariton gas is even "hotter" than the phonon bath itself. This surprising result suggests that introducing a nonequilibrium character to a coolant fluid might constitute a useful resource to enhance its performance.

In this work we have shown, as an experimental proof of principle, that polaritonic cooling of a semiconductor $\mathrm{MC}$ works and presents unusual properties with respect to stateof-the-art methods of optical cooling in solids. First, owing to the specific polaritonic density of states, which allows removing low energy thermal acoustic phonons, cooling can be achieved at arbitrarily low cryostat temperature. Second, polaritonic cooling involves a new mechanism referred to as fast cooling that is unique to polaritons, and opens up an experimental window on nonequilibrium thermodynamics of phonons. Finally, we have shown that polariton fluids constitute an experimental model system to study the heat transport properties between a thermal bath and a nonequilibrium fluid.

E. D. and S. K. have contributed equally to this work. All the authors acknowledge the support by the ERC StG Contract No. 258608. M.R., A. M., S.D., S. K., A. B., Y. L., and E. D. wish to thank M. Wouters, I. Carusotto, L. S. Dang, J. Kasprzak, J. Bloch, and A. Amo for inspiring discussions. Technical support by C. Bouchard and L. Del-Rey is warmly acknowledged.

[1] Special issue on Laser Cooling and Trapping of Atoms, edited by S. Chu and C. Wieman [J. Opt. Soc. Am. B 6, 2020 (1989)].

[2] K. C. Neuman and S. M. Block, Rev. Sci. Instrum. 75, 2787 (2004).

[3] S. Gigan, H. R. Böhm, M. Paternostro, F. Blaser, G. Langer, J. B. Hertzberg, K. C. Schwab, D. Bäuerle, M. Aspelmeyer, and A. Zeilinger, Nature (London) 444, 67 (2006).

[4] O. Arcizet, P. F. Cohadon, T. Briant, M. Pinard, and A. Heidmann, Nature (London) 444, 71 (2006).

[5] J. Chan, T. Alegre, A. Safavi-Naeini, J. Hill, A. Krause, S. Groblacher, M. Aspelmeyer, and O. Painter, Nature (London) 478, 89 (2011).

[6] P. Pringsheim, Z. Phys. A 57, 739 (1929).

[7] M. Sheik-Bahae and R. I. Epstein, Nat. Photonics 1, 693 (2007).

[8] D. V. Seletskiy, S. D. Melgaard, R. I. Epstein, A. Di Lieto, M. Tonelli, and M. Sheik-Bahae, Opt. Express 19, 18229 (2011).

[9] G. Rupper, N. H. Kwong, and R. Binder, Phys. Rev. Lett. 97, 117401 (2006).
[10] M. Sheik-Bahae and R. I. Epstein, Phys. Rev. Lett. 92, 247403 (2004).

[11] H. Gauck, T. H. Gfroerer, M. J. Renn, E. A. Cornell, and K. A. Bertness, Appl. Phys. A 64, 143 (1997).

[12] E. Finkeissen, M. Potemski, P. Wyder, L. Vina, and G. Weimann, Appl. Phys. Lett. 75, 1258 (1999).

[13] J. Zhang, D. Li, R. Chen, and Q. Xiong, Nature (London) 493, 504 (2013).

[14] D. V. Lang and C. H. Henry, Phys. Rev. Lett. 35, 1525 (1975).

[15] C. Weisbuch, M. Nishioka, A. Ishikawa, and Y. Arakawa, Phys. Rev. Lett. 69, 3314 (1992).

[16] A. Fainstein, B. Jusserand, and V. Thierry-Mieg, Phys. Rev. Lett. 78, 1576 (1997).

[17] G. Rozas, A. E. Bruchhausen, A. Fainstein, B. Jusserand, and A. Lemaitre, Phys. Rev. B 90, 201302(R) (2014).

[18] See Supplemental Material at http://link.aps.org/ supplemental/10.1103/PhysRevLett.114.186403, which includes Refs. [19-26,31], for more details on the experimental and theoretical aspects of this work.

[19] W. Langbein, I. Shelykh, D. Solnyshkov, G. Malpuech, Yu. Rubo, and A. Kavokin, Phys. Rev. B 75, 075323 (2007).

[20] A. Kavokin, G. Malpuech, and M. Glazov, Phys. Rev. Lett. 95, 136601 (2005).

[21] C. Leyder, M. Romanelli, J. P. Karr, E. Giacobino, T. C. H. Liew, M. M. Glazov, A. V. Kavokin, G. Malpuech, and A. Bramati, Nat. Phys. 3, 628 (2007).

[22] F. Tassone, C. Piermarocchi, V. Savona, A. Quattropani, and P. Schwendimann, Phys. Rev. B 53, R7642 (1996).

[23] C. Piermarocchi, F. Tassone, V. Savona, A. Quattropani, and P. Schwendimann, Phys. Rev. B 53, 15834 (1996).

[24] M. Cardona and N. E. Christensen, Phys. Rev. B 35, 6182 (1987).

[25] S. K. Zhang, H. Lu, W. B. Wang, B. B. Das, N. Okoye, M. Tamargo, and R. R. Alfano, J. Appl. Phys. 101, 023111 (2007).

[26] S. Klembt, K. Frank, G. Qian, T. Klein, A. Rosenauer, D. Hommel, and C. Kruse, J. Cryst. Growth 378, 270 (2013).

[27] T. Amand and X. Marie, Exciton Spin Dynamics in Semiconductor Quantum Wells in Spin Physics in Semiconductors, edited by M. I. Dyakonov (Springer, Berlin, 2008).

[28] K. Sebald, M. Seyfried, S. Klembt, S. Bley, A. Rosenauer, D. Hommel, and C. Kruse, Appl. Phys. Lett. 100, 161104 (2012).

[29] M. Umlauff, J. Hoffmann, H. Kalt, W. Langbein, J. M. Hvam, M. Scholl, J. Sollner, M. Heuken, B. Jobst, and D. Hommel, Phys. Rev. B 57, 1390 (1998).

[30] G. Rozas, M. F. Pascual Winter, B. Jusserand, A. Fainstein, B. Perrin, E. Semenova, and A. Lemaitre, Phys. Rev. Lett. 102, 015502 (2009).

[31] S. Rudin, T. L. Reinecke, and B. Segall, Phys. Rev. B 42, 11218 (1990).

[32] A. Trichet, L. Sun, G. Pavlovic, N. A. Gippius, G. Malpuech, W. Xie, Z. Chen, M. Richard, and Le Si Dang, Phys. Rev. B 83, 041302(R) (2011). 